\title{
Regressive Changes of the Myocardial Sleeves in Elderly Victims of Sudden Bathtub Death
}

\author{
Fumiko Satoh*, Yoshihisa Seto, Akio Tsuboi, Motoki Osawa \\ Department of Forensic Medicine, Tokai University School of Medicine, Isehara, Japan \\ Email: ${ }^{\text {fumiko-s@is.icc.u-tokai.ac.jp }}$
}

Received 9 February 2015; accepted 3 April 2015; published 7 April 2015

Copyright (C) 2015 by authors and Scientific Research Publishing Inc.

This work is licensed under the Creative Commons Attribution International License (CC BY). http://creativecommons.org/licenses/by/4.0/

\section{(c) (7) Open Access}

\begin{abstract}
In Japan, most sudden deaths occurring during bathing happen in the winter, and predominantly to elderly people. One can infer a relation to physical conditions that are specific to aging. Atrial fibrillation, an arrhythmia, increases with age. This study examined histological changes in the pulmonary vein myocardial sleeves of sudden bathtub death victims and compared them with those of control individuals. We investigated 35 sudden deaths that occurred during bathing and 34 accidental deaths or deaths caused by diseases unrelated to cardiopathies. Pulmonary veins were excised cross-sectionally from the hilar side to the venoatrial junction. Then they were stained with hematoxylin and eosin, resorcin-fuchsin van Gieson, and Congo-red stains. Amyloid deposits in the pulmonary vein myocardial sleeves, as well as the range and severity of scarring, were graded microscopically on a scale of 0 -3. In the sudden bathtub death victims, severe scarring was found in the myocardial sleeves of the four pulmonary veins (mean score, 2.0), which was significantly different $(p<0.05)$ from the control subjects (mean score, 1.4). Cardiomegaly was found in 28 out of the autopsied individuals. In subjects with cardiomegaly, the mean value of pulmonary vein myocardial sleeve scarring was 2.1. In subjects without cardiomegaly, the mean value was 1.8 . Comparison revealed that cardiomegaly was associated significantly with scarring progression and degeneration of the myocardial sleeves. Scarring of the pulmonary vein myocardial sleeves was more advanced in victims of sudden bathtub death than in controls without heart disease. Elderly people with scarring of the pulmonary vein myocardial sleeves are likely to develop degenerative variations in their intra-atrial excitation conduction. These results demonstrate that taking hot baths might induce supraventricular arrhythmias such as atrial fibrillation.
\end{abstract}

\section{Keywords}

Sudden Bathtub Death, Autopsy, Pulmonary Veins, Myocardial Sleeves, Atrial Fibrillation, Scarring

\footnotetext{
${ }^{*}$ Corresponding author.
}

How to cite this paper: Satoh, F., Seto, Y., Tsuboi, A. and Osawa, M. (2015) Regressive Changes of the Myocardial Sleeves in Elderly Victims of Sudden Bathtub Death. Forensic Medicine and Anatomy Research, 3, 57-65. 


\section{Introduction}

Japanese people have a unique mode of bathing. A bather is immersed up to the shoulders for approximately 10 - $30 \mathrm{~min}$ in a bathtub that has been filled with hot water $\left(40^{\circ} \mathrm{C}-42^{\circ} \mathrm{C}\right)$. Consequently, in Japan, sudden deaths of elderly people during bathing constitute an important social issue. The immediate cause of death in most cases is drowning caused by the aspiration of hot water. This manner of death, designated as the "dead in hot bathtub phenomenon" [1], becomes increasingly common during the winter: between November and March. The mechanisms underlying sudden bathtub death have not yet been fully elucidated. Sudden bathtub deaths predominantly affect elderly people. Therefore, this phenomenon is suspected of being associated with physical conditions specific to aging. This study specifically examines a putative connection to age-related changes to the cardiovascular system.

Chiba et al. reported the development of arrhythmias, such as supraventricular extrasystole, atrial fibrillation, ventricular extrasystole, and ventricular tachycardia, in elderly subjects during bathing in the winter [2]. Based on various parameters including electrocardiographic data, the study participants were regarded as demonstrating changes that placed them at high risk. Therefore, when they entered a hot bath during the cold season, the effects of hydrostatic pressure generated physical compensatory effects, which might have caused coronary artery stenosis. The stenosis might have a particularly strong effect on elderly people with reduced respiratory function. When a diastolic overload occurs rapidly in the right atrium, attributable to an increase in venous return resulting from the increased hydrostatic pressure during bathing, the resulting dilation of the right atrium is likely to cause morphological changes in the tricuspid annulus. These effects are likely to cause similar changes in the atrioventricular node, which is located near the septal leaflet of the tricuspid valve. Such changes might induce supraventricular arrhythmia. When a sudden, bath-related increase in blood pressure and heart rate occurs in conjunction with atrial fibrillation or supraventricular arrhythmia, a person might experience dizziness or unconsciousness. For a person immersed in a bathtub, fatal aspiration of water might occur.

Atrial fibrillation increases with advancing age. The prevalence of atrial fibrillation is reported to begin increasing at approximately 40 years of age. For people in their fourth decade of life, the prevalence is $2.3 \%$, rising to $5.9 \%$ in individuals who are 65 years old or older [3]. In general, in addition to heart disease (such as mitral stenosis, mitral insufficiency, and atrial septal defects), which adds an additional load on the atrium, other causes of atrial fibrillation include hypertension, endocrine disorders, stress, and alcohol use. Typically, these factors mutually interact in a complex manner [3] [4].

Haïssaguerre et al. reported previously that, in elderly people and patients with atrial fibrillation caused by organic heart disease, most ectopic stimulations arise from the pulmonary veins [5] [6]. In regions surrounding the pulmonary veins in humans and animals, some atrial muscles from the left atrium have a sleeve-like elongated shape. Inflammation and pressure loads might engender fibrosis, extension, and thinning of the atrial wall, enhancing the normal degenerative histological changes that begin after birth. The atrial muscle sleeves of the pulmonary veins are continuous with atrial muscles. They also change with age. Consequently, fibrosis of the myocardial sleeves occurs in addition to disordered arrangement of the muscle sleeve margins. Their structural and electrical reshaping is believed to progress with age, engendering the development of atrial fibrillation [7][9].

Our previous report on arrhythmias involving fibrosis and degeneration of the myocardial sleeves showed that, compared with patients with no history of atrial fibrillation, those with a history of atrial fibrillation exhibited significantly greater degeneration and disturbances of the muscle sleeve margins [10]. Conduction can be blocked because of the presence of pulmonary vein myocardial sleeve scarring, causing atrial fibrillation.

A considerable number of victims of sudden bathtub death had no history of disease. In most cases, data related to electrocardiographic abnormalities are not available. Based on autopsy results, morphological demonstration of electrophysiological cardiac abnormalities was also not feasible. Therefore, the most suitable method is believed to estimate electrocardiographic abnormalities through detailed examinations of cardiac morphological changes.

This study was conducted to elucidate the morphological changes causing atrial fibrillation, and to ascertain the changes in pulmonary vein myocardial sleeves observed in autopsy specimens from sudden bathtub death victims, compared with those in control individuals.

\section{Subjects and Methods}

The study examined 69 heart specimens, including the four pulmonary veins, obtained during autopsies con- 
ducted from January 2007 through December 2008. The 35 people who died of sudden bathtub death showed signs of drowning, but the cause of drowning was unknown in each case. The 34 control subjects were autopsy specimens from individuals who had died of diseases or accidents, but who did not have a history of heart disease such as arrhythmia. The selection of the subjects was adjusted by age to avoid differences in clinical characteristics between both groups.

To excise the pulmonary veins, cardiac tissue incisions were extended to the hilar side. Each heart was excised along with its pulmonary veins. Gauze was inserted into the pulmonary veins to maintain the specimen shape. The specimens were fixed in $10 \%$ buffered formalin. Figure 1 schematically depicts the objective pulmonary veins in the heart. The four pulmonary veins (the left and right superior pulmonary veins, and the left and right inferior pulmonary veins) were cut in a cross-sectional manner from the hilar side to the venoatrial junction at intervals of $4-5 \mathrm{~mm}$. The slices were stained with hematoxylin and eosin, resorcin-fuchsin van Gieson, and Congo-red stains.

The amyloid deposits in the myocardial sleeves of the pulmonary veins, as well as the range and severity of the scarring, were graded after masking the personal information and history of the subjects. The grading was done using a scale of 0-3, based on the following criteria: isolated atrial amyloid deposits were graded as 0 (not present), 1 (occasional fine fibers around cardiomyocytes and/or in the walls of small intramyocardial vessels, mostly in the inner layers of the sleeve), 2 (more frequent fibers throughout the entire thickness of the sleeve, and 3 (dense network of fibers and/or solid foci). Scarring was graded as 0 (absent), 1 (isolated foci of fibrosis, affecting only a part of the sleeve and/or mild diffuse interstitial fibrosis), 2 (isolated foci of fibrosis affecting the entire thickness of the sleeve and/or moderately severe diffuse interstitial fibrosis), and 3 (multiple confluent foci affecting the entire sleeve thickness and/or severe scaring completely replacing the myocardium). Cardiomegaly was assessed as heart weight $>400 \mathrm{~g}$, Sato's cardiac index [11] $>2.5$ points, and left ventricular wall thickness measuring $\geq 1.5 \mathrm{~cm}$.

The presence or absence of statistically significant differences was tested using Fisher's exact test. A $p$-value $<0.05$ was inferred as a statistically significant difference.

\section{Results}

The mean age of the 34 sudden bathtub death victims (18 male, 16 female) was $74.5 \pm 11.7$ years. The mean age of the 35 control individuals (26 male, 9 female) was $75.0 \pm 13.0$ years. Among the sudden bathtub death cases, only two individuals had a history of atrial fibrillation (Table 1), but the immediate cause of death in all cases was drowning. In the control group, 19 individuals died because of traumatic injuries; 16 died because of endogenous, non-cardiac-related causes.

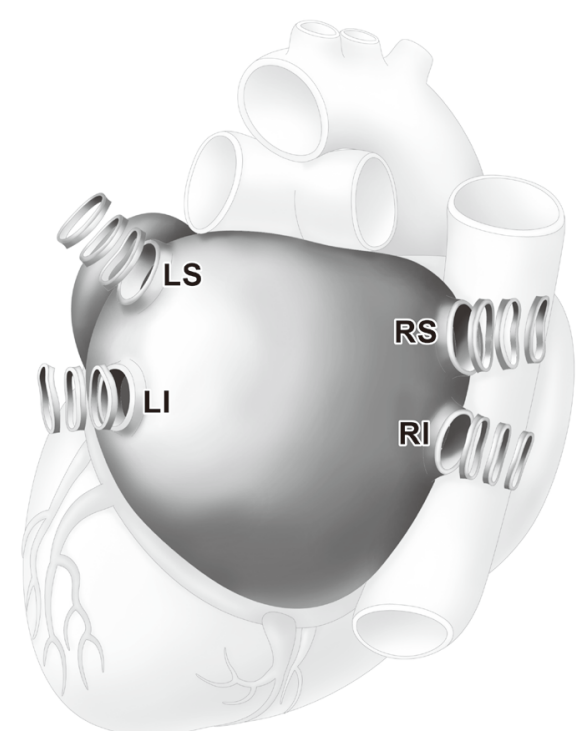

Figure 1. Schema showing the pulmonary vein (PV) locations: LS = left superior $\mathrm{PV}, \mathrm{LI}=$ left inferior PV, RS = right superior PV, RI = right inferior PV. 
Table 1. Medical history and the socio-demographic characteristics of the patients of the bathtub sudden death $(n=35)$.

\begin{tabular}{cc}
\hline Medical history & Number (\%) \\
Hypertension & $5(14 \%)$ \\
Diabetes mellitus & $2(6 \%)$ \\
Atrial fibrillation & $2(6 \%)$ \\
Sick sinus syndrome & $1(3 \%)$ \\
Chronic rheumatoid arthritis & $1(3 \%)$ \\
Angina pectoris & $1(3 \%)$ \\
Cerebral infarction & $1(3 \%)$ \\
Chronic renal failure & $1(3 \%)$ \\
Hepatic failure & $1(3 \%)$ \\
Duodenal ulcer & $1(3 \%)$ \\
Gastric ulcer & $1(3 \%)$ \\
Parkinson's disease & $1(3 \%)$ \\
No history & $19(54 \%)$ \\
\hline President of a company & Number (\%) \\
Electric constructor & $31(88.5)$ \\
\hline Unemployed & $2(5.7)$ \\
\hline Oofice worker & $1(2.9)$ \\
\hline
\end{tabular}

The histological examination, involving amyloidosis and scarring grading, was performed separately by two pathologists blinded to the clinical data and autopsy findings. The results obtained by the two pathologists were not greatly different, but when differences arose, the results were discussed. A consensus was reached in each case.

The myocardial sleeves are shaped by left atrial myocardium extending over the pulmonary veins, covering them for a variable distance towards the pulmonary hilus and even beyond it. The sleeves are always positioned on the adventitial side of the pulmonary veins, separated from the media by a fibrofatty layers (Figure 2). The histopathologic evaluation revealed that the average degree of myocardial sleeve scarring was 2.0 in the sudden bathtub death group, and 1.4 in the control group; this difference was significant (Table 2). For the sudden bathtub death cases and the controls, no significant difference was found in the extent of scarring of the four pulmonary veins.

Scaring of the myocardial sleeves was universal, present at least mildly in all 273 sleeves. In the mild cases, intercellular fibrosis affected only an isolated muscle bundle. In the advanced cases, the whole thickness was collagenised and the sleeve become thinner, most markedly at the periphery; the fibrosed fingerlike projections of its myocardium acquired a winding shape (Figure 3).

In the myocardial sleeves of pulmonary veins, amyloid deposition was present in 55 hearts (79.7\%) in a total of 146 sleeves (53.4\%) (Figure 4). The mean pulmonary vein myocardial sleeve amyloid deposition score was 1.0 in the sudden bathtub death group and 0.8 in the control group. The difference between the groups was not significant (Table 3). The sudden bathtub death hearts showed no abnormal findings such as bleeding or fibrosis in the atrioventricular node.

Cardiomegaly was present in 16 of the sudden bathtub death hearts and in 12 of the control organs; 41 hearts from the two groups exhibited no cardiomegaly. The mean pulmonary vein myocardial sleeve scarring score was 2.1 in individuals with cardiomegaly and 1.8 in individuals without cardiomegaly. These findings demonstrated that the scarring, fibrosis, and degeneration of the pulmonary vein myocardial sleeves were significantly more advanced in individuals with cardiomegaly (Table 4). 


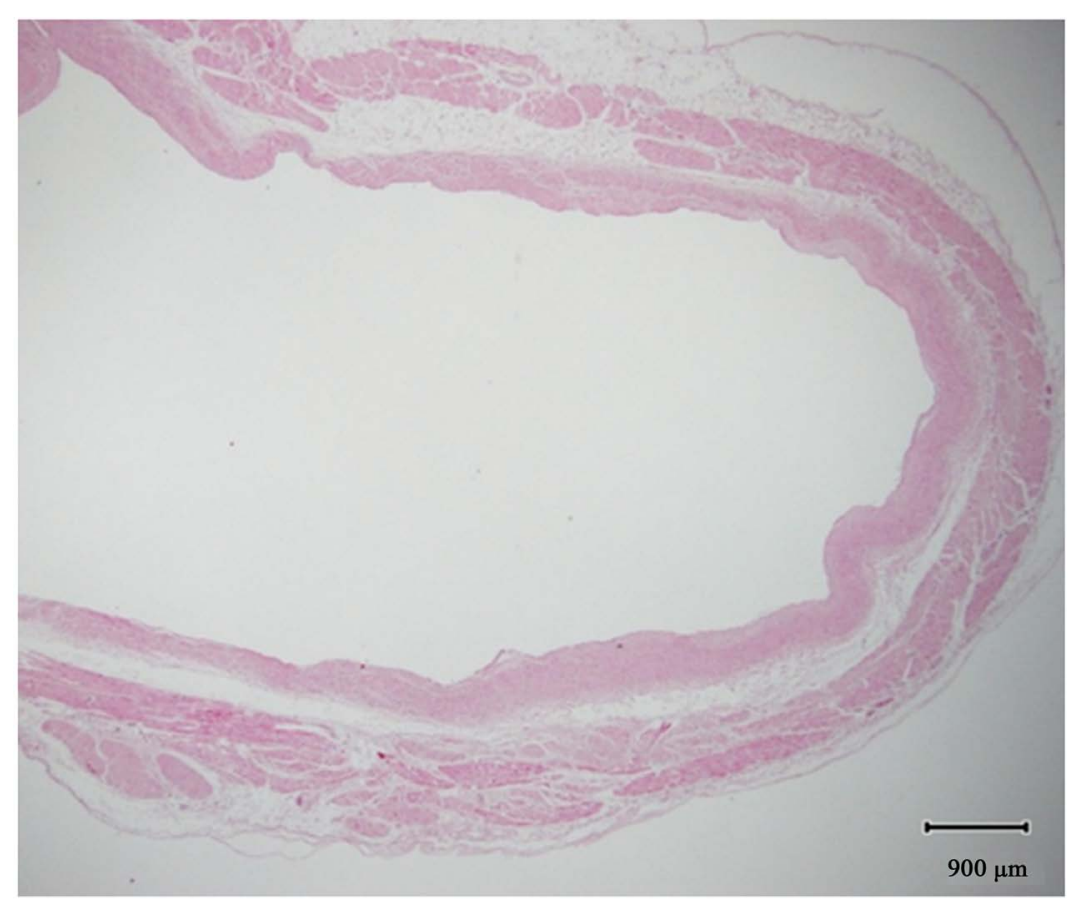

Figure 2. Segment of a well-developed pulmonary vein myocardial sleeve.

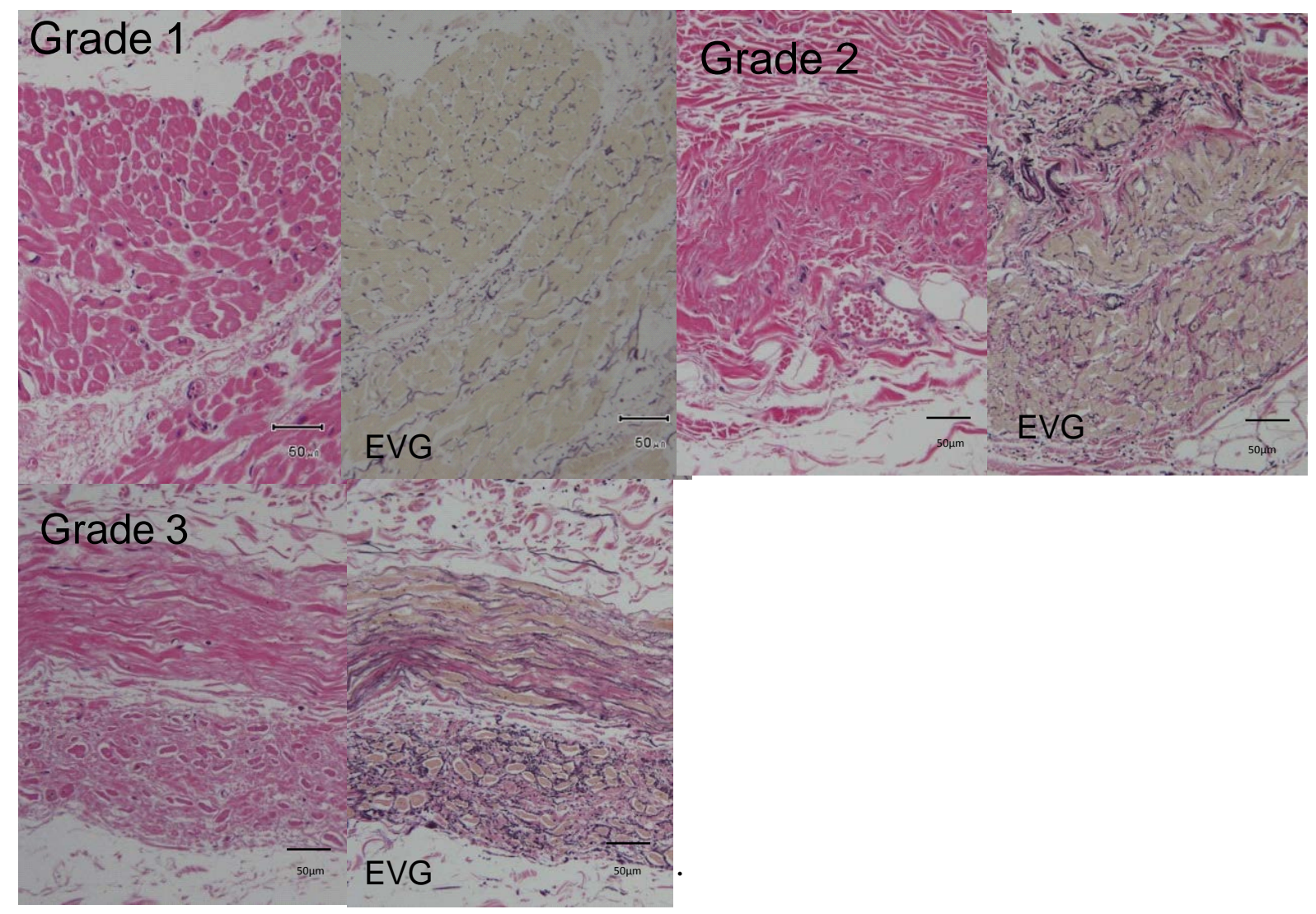

Figure 3. Myocardial sleeve scar grading (hematoxylin and eosin staining [HE, left image of each pair] or resorcin-fuchsin van Gieson staining [EVG, right image of each pair]). Grading of scarring: Grade $0=$ absent, Grade $1=$ mild diffuse interstitial fibrosis, Grade 2 = moderately diffuse interstitial fibrosis, Grade 3 = severely diffuse interstitial fibrosis. 


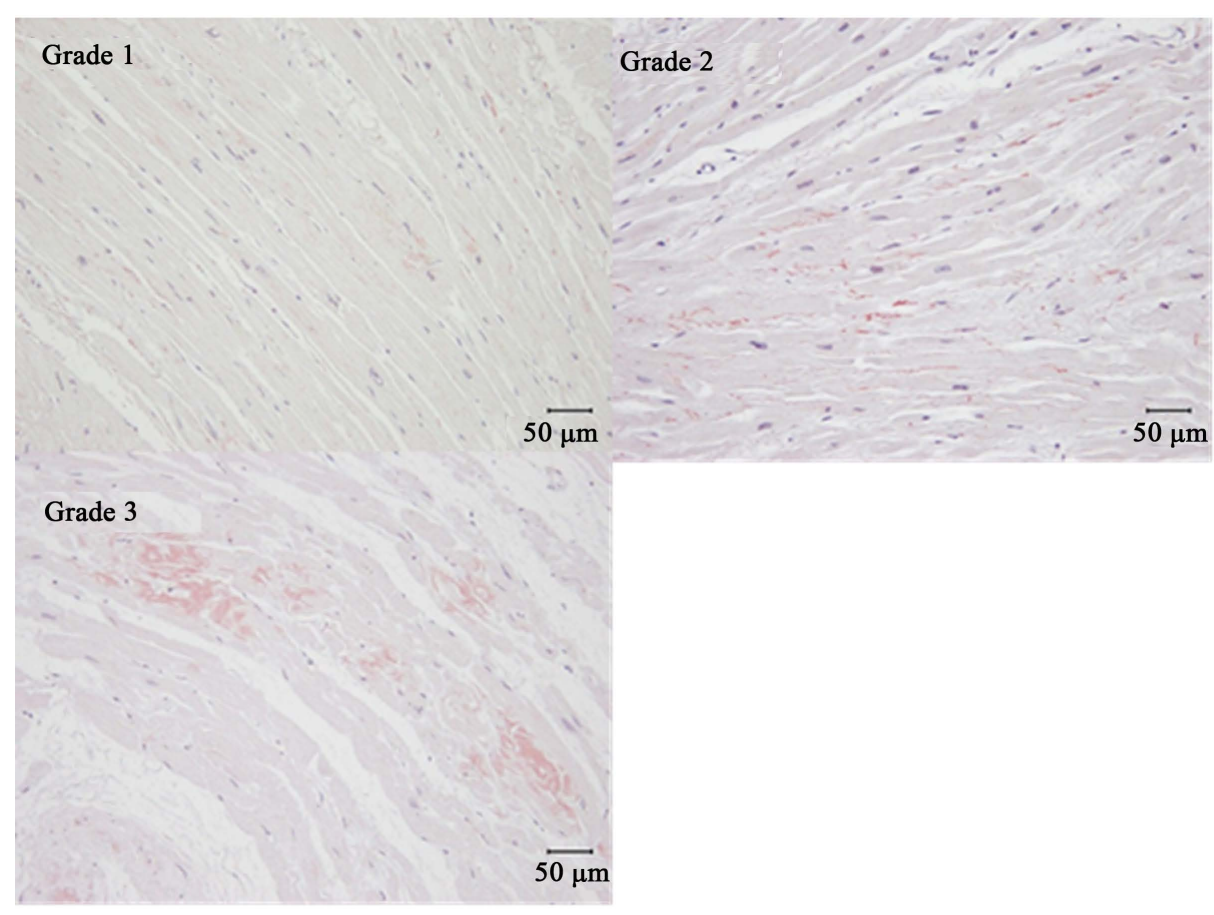

Figure 4. Myocardial sleeve amyloid deposition grading (Congo-red staining). Grading of amyloid deposition: Grade 0 = not present, Grade 1 = occasional fine fibers, Grade 2 = more frequent fibers in the overall thickness, Grade 3 = dense network of fibers.

Table 2. Degree of pulmonary vein myocardial sleeve scarring.

\begin{tabular}{|c|c|c|c|c|c|c|c|c|c|c|c|c|c|}
\hline & \multicolumn{2}{|c|}{ Grade 0} & \multicolumn{2}{|c|}{ Grade 1} & \multicolumn{2}{|c|}{ Grade 2} & \multicolumn{2}{|c|}{ Grade 3} & \multicolumn{2}{|c|}{ Subtotal } & \multicolumn{2}{|c|}{ Average } & \multirow{2}{*}{$p$} \\
\hline & $\mathrm{BD}$ & C & $\mathrm{BD}$ & $\mathrm{C}$ & $\mathrm{BD}$ & $\mathrm{C}$ & $\mathrm{BD}$ & $\mathrm{C}$ & $\mathrm{BD}$ & C & $\mathrm{BD}$ & $\mathrm{C}$ & \\
\hline LS & 0 & 0 & 6 & 18 & 21 & 13 & 6 & 2 & 33 & 33 & 1.9 & 1.52 & $<0.05$ \\
\hline LI & 0 & 0 & 8 & 16 & 16 & 10 & 11 & 6 & 32 & 32 & 2.1 & 1.69 & $<0.05$ \\
\hline RS & 0 & 0 & 8 & 16 & 16 & 13 & 11 & 4 & 33 & 33 & 1.9 & 1.63 & $<0.05$ \\
\hline RI & 0 & 0 & 11 & 23 & 17 & 11 & 11 & 1 & 35 & 35 & 1.7 & 1.37 & $<0.05$ \\
\hline$\Sigma$ & 0 & 0 & 33 & 76 & 47 & 47 & 32 & 13 & 137 & 136 & 2 & 1.44 & \\
\hline
\end{tabular}

BD, bathtub drowning cases ( $n=34$ ); C, control cases ( $n=35)$; LS, left superior; LI, left inferior; RS, right superior; RI, right inferior.

Table 3. Isolated atrial amyloid in pulmonary vein myocardial sleeves.

\begin{tabular}{|c|c|c|c|c|c|c|c|c|c|c|c|c|c|}
\hline & \multicolumn{2}{|c|}{ Grade 0} & \multicolumn{2}{|c|}{ Grade 1} & \multicolumn{2}{|c|}{ Grade 2} & \multicolumn{2}{|c|}{ Grade 3} & \multicolumn{2}{|c|}{ Subtotal } & \multicolumn{2}{|c|}{ Average } & \multirow{2}{*}{$p$} \\
\hline & $\mathrm{BD}$ & $\mathrm{C}$ & $\mathrm{BD}$ & $\mathrm{C}$ & $\mathrm{BD}$ & $\mathrm{C}$ & $\mathrm{BD}$ & $\mathrm{C}$ & $\mathrm{BD}$ & $\mathrm{C}$ & $\mathrm{BD}$ & $\mathrm{C}$ & \\
\hline LS & 21 & 12 & 9 & 15 & 1 & 5 & 2 & 1 & 33 & 33 & 0.5 & 0.85 & 0.06 \\
\hline LI & 20 & 14 & 10 & 9 & 4 & 6 & 1 & 3 & 35 & 32 & 0.6 & 0.94 & 0.53 \\
\hline RS & 16 & 10 & 9 & 11 & 6 & 8 & 4 & 4 & 35 & 33 & 0.9 & 1.18 & 0.64 \\
\hline RI & 13 & 16 & 10 & 12 & 5 & 5 & 4 & 2 & 32 & 35 & 1 & 0.8 & 0.83 \\
\hline$\Sigma$ & 70 & 55 & 38 & 47 & 16 & 24 & 13 & 10 & 135 & 136 & 1 & 0.75 & \\
\hline
\end{tabular}

BD, bathtub drowning cases ( $n=34)$; C, control cases $(n=35)$; LS, left superior; LI, left inferior; RS, right superior; RI, right inferior. 
Table 4. Association of cardiomegaly with myocardial sleeve scarring.

\begin{tabular}{cccc}
\hline Cardiomegaly & Number & Average grade of sleeve scaring & $p$ \\
\hline Yes & 28 & 2.11 & $<0.05$ \\
No & 41 & 1.77 & \\
\hline
\end{tabular}

\section{Discussion}

Sudden bathtub deaths occur often in Japan, although the phenomenon is rare in the United States and in European countries [1], probably because of the unique bathing behaviors of Japanese people. Several theories exist in relation to the causes of sudden bathtub deaths. Nevertheless, the pathogenesis of the condition has not been fully elucidated to date. The three major theories related to the mechanism of sudden bathtub death are hyperthermia, transient ischemic attacks, and critical arrhythmias.

Yoshioka et al. reported previously that when an elderly subject bathed in hot water at a temperature of $41^{\circ} \mathrm{C}$ for $3 \mathrm{~min}$, the person's body temperature rose to $38^{\circ} \mathrm{C}-39^{\circ} \mathrm{C}$, taking approximately $10 \mathrm{~min}$ to return to normal after bathing [12]. Elderly people are believed to have disturbed autonomic functions. As a result, they often have sweating and cutaneous vasodilatory response dysfunctions. Such autonomic dysfunctions are therefore believed to make them more susceptible to hyperthermia. Consequently, they are more prone to a loss of consciousness during a hot bath, and might die of heat stroke-related drowning in the bathtub.

Shigeomi et al. previously reported blood pressures and pulse rates of elderly subjects ( $\geq 60$ years old) before bathing, in the dressing room, immediately after entering the bath, during bathing, and after exiting the bath. They found that, in the winter, the systolic blood pressures of the individuals increased while in the dressing room. Then, two minutes after entering the bath, their systolic blood pressure decreased significantly compared to that measured before bathing [13]. When these blood pressure fluctuations are of high amplitude, the individual is at increased risk of experiencing a transient ischemic attack. If a transient ischemic attack occurs while the subject is bathing, drowning is likely.

Chiba et al. reported that when elderly people bathe for five minutes in hot water at a temperature of $41^{\circ} \mathrm{C}$ in the winter, they experience increased oxygen consumption, peripheral vascular resistance, and cardiac output. The electrocardiograms of 12 out of 20 elderly subjects showed arrhythmias while bathing, such as supraventricular extrasystole, atrial fibrillation, ventricular extrasystole, and ventricular tachycardia. These findings suggest that bathing induces cardiopulmonary function fluctuations. Again, if a loss of consciousness occurs as a result of an arrhythmia during bathing, then the risk of death caused by drowning is increased [2].

Autopsies of sudden bathtub death victims were surveyed based on the deceased person's age, sex, bathing location, month of occurrence, cause of death, and presence or absence of findings suggesting drowning. Results showed that the victims had a mean age of 71.8 years. Most deaths occurred during the winter: between November and March. Among the causes of death, ischemic heart disease accounted for 31.6\%, cardiomegaly accounted for $29.9 \%$, and high blood alcohol levels accounted for $16.7 \%$. However, the autopsy findings usually revealed no evidence of any other disease except age-related arteriosclerosis. In a considerable number of cases, isolated findings of drowning were found in the lungs. Toxicology results were negative [1].

In some cases of sudden bathtub death, the cause of the unconsciousness in the bathtub remained unknown, even after the autopsy. From autopsy findings, we inferred that in some victims, arrhythmia might have caused a loss of consciousness and drowning.

For this investigation of the causes of sudden bathtub deaths, we examined the histological changes in the myocardium at the level of the myocardial sleeves of the pulmonary veins, which are regarded as the origins of atrial fibrillation. We compared autopsy cases of sudden bathtub death with autopsy investigations of control hearts without heart disease. The findings showed that the pulmonary vein myocardial sleeves demonstrated significantly more fibrosis and scar formation in victims of sudden bathtub death than did those of the controls.

Previous reports have described scarring, fibrosis, and histological discontinuity of the myocardial sleeves as progressive. They are reportedly most severe in autopsy cases with atrial fibrillation [7]. Reshaping of the muscle layers of the pulmonary vein myocardial sleeves is associated with aging. Their degeneration results from the infiltration of inflammatory cells and subsequent collagen fiber formation [14]. These changes are attributable to increased atrial tension caused by increased pressure on the left atrium.

During the onset of atrial fibrillation, functional and histological changes in the atrial muscles and myocardial 
sleeves cause variations in nerve excitation and conduction, leading to the formation of multiple excitation waves propagating in different directions. Each excitation wave shows a swirling, known as "reentry," while exciting the atrium in such a way as to fill the excitation gap. This causes the development of the so-called spiral entry, which is believed to trigger atrial fibrillation [15]-[17]. Elevated left atrial pressure and enlargement of the left atrial and pulmonary vein diameters have been reported from a clinical study of atrial fibrillation in patients, without underlying disease. The incidence of atrial fibrillation increases with age. Therefore, it is likely to be caused by the age-related degeneration, fibrosis, and failure of the myocardial sleeves [18].

Table 4 shows the severity of myocardial sleeve fibrosis and scarring among the 28 cases of cardiomegaly and the 41 cases without cardiomegaly. In the presence of cardiomegaly, myocardial sleeve fibrosis was severe, and showed a significant difference compared with that found in individuals without cardiomegaly. Cardiomegaly was found in 17 of the 35 sudden bathtub death victims. The pressure overload attributable to cardiomegaly was inferred as a cause of the pulmonary vein myocardial sleeve scarring and fibrosis. However, scarring of the pulmonary vein myocardial sleeves was also found in some individuals without cardiomegaly, suggesting the need for additional investigation.

Results of this study showed that, compared with controls without heart disease, the sudden bathtub death victims showed increased scarring of the pulmonary vein myocardial sleeves. This result suggests that these regions might be the foci for the development of supraventricular arrhythmias such as asymptomatic atrial fibrillation. Results of this study suggest that, among elderly victims of sudden bathtub death, deaths in some cases might be attributable to asymptomatic atrial fibrillation.

\section{Conclusion}

We compared autopsy cases of sudden bathtub death with autopsy investigations of control hearts without heart disease. The findings showed that the pulmonary vein myocardial sleeves demonstrated significantly more fibrosis and scar formation in victims of sudden bathtub death than did those of the controls. Reshaping of the muscle layers of the pulmonary vein myocardial sleeves is associated with aging. Results of this study suggest that, among elderly victims of sudden bathtub death, deaths in some cases might be attributable to asymptomatic atrial fibrillation.

\section{Acknowledgements}

This study was funded by a Grant-in-Aid for Scientific Research (Grant No. 19590680).

\section{References}

[1] Satoh, F., Osawa, M., Hasegawa, I., Seto, Y. and Tsuboi, A. (2013) "Dead in Hot Bathtub” Phenomenon. Accidental Drowning or Natural Disease? American Journal of Forensic Medicine and Pathology, 34, 164-168. http://dx.doi.org/10.1097/PAF.0b013e31828d68c7

[2] Chiba, T., Yamauchi, M., Nishida, N., Kaneko, T., Yoshizaki, K. and Yoshioka, N. (2005) Risk Factors of Sudden Death in the Japanese Hot Bath in the Senior Population. Forensic Science International, 149, 151-158. http://dx.doi.org/10.1016/j.forsciint.2004.04.085

[3] Benjamin, E.J., Wolf, P.A., D’Agostino, R.B., Silbershatz, H., Kannel, W.B. and Levy, D. (1998) Impact of Atrial Fibrillation on the Risk of Death: The Framingham Heart Study. Circulation, 98, 946-952. http://dx.doi.org/10.1161/01.CIR.98.10.946

[4] Lloyd-Jones, D.M., Wang, T.J., Leip, E.P., Larson, M.G., Levy, D., Vasan, R.S., D’Agostino, R.B., Massaro, J.M., Beiser, A., Wolf, P.A. and Benjamin, E.J. (2004) Lifetime Risk for Development of Atrial Fibrillation: The Framingham Heart Study. Circulation, 110, 1042-1046. http://dx.doi.org/10.1161/01.CIR.0000140263.20897.42

[5] Haissaguerre, M., Jaïs, P., Shah, D.C., Takahashi, A., Hocini, M., Quiniou, G., Garrigue, S., Le Mouroux, A., Le Métayer, P. and Clémenty, J. (1998) Spontaneous Initiation of Atrial Fibrillation by Ectopic Beats Originating in the Pulmonary Veins. New England Journal of Medicine, 339, 659-666. http://dx.doi.org/10.1056/NEJM199809033391003

[6] Haissaguerre, M., Jaïs, P., Shah, D.C., Arentz, T., Kalusche, D., Takahashi, A., Garrigue, S., Hocini, M., Peng, J.T. and Clémenty, J. (2000) Catheter Ablation of Chronic Atrial Fibrillation Targeting the Reinitiating Triggers. Journal of Cardiovascular Electrophysiology, 11, 2-10. http://dx.doi.org/10.1111/j.1540-8167.2000.tb00727.x

[7] Steiner, I., Hájková, P., Kvasnička, J. and Kholová, I. (2006) Myocardial Sleeves of Pulmonary Veins and Atrial Fi- 
brillation: A Postmortem Histopathological Study of 100 Subjects. Virchows Archiv, 449, 88-95. http://dx.doi.org/10.1007/s00428-006-0197-2

[8] Fynn, S.P. and Kalman, J.M. (2004) Pulmonary Veins: Anatomy, Electrophysiology, Tachycardia, and Fibrillation. Pacing and Clinical Electrophysiology, 27, 1547-1559. http://dx.doi.org/10.1111/j.1540-8159.2004.00675.X

[9] Hsieh, M.H., Tai, C.T., Tsai, C.F., Yu, W.C., Lee, S.H., Lin, Y.K., Ding, Y.A., Chang, M.S. and Chen, S.A. (2000) Pulmonary Vein Electrogram Characteristics in Patients with Focal Sources of Paroxysmal Atrial Fibrillation. Journal of Cardiovascular Electrophysiology, 11, 953-959. http://dx.doi.org/10.1111/j.1540-8167.2000.tb00166.x

[10] Saito, T., Waki, K. and Becker, A.E. (2000) Left Atrial Myocardial Extension onto Pulmonary Veins in Humans: Anatomic Observations Relevant for Atrial Arrhythmias. Journal of Cardiovascular Electrophysiology, 11, 888-894. http://dx.doi.org/10.1111/j.1540-8167.2000.tb00068.x

[11] Hitosugi, M., Takatsu, A., Kinugasa, Y. and Takao, H. (1999) Estimation of Normal Heart Weight in Japanese Subjects: Development of a Simplified Normal Heart Weight Scale. Legal Medicine, 1, 80-85. http://dx.doi.org/10.1016/S1344-6223(99)80017-0

[12] Yoshioka, N. (2008) Sudden Death in the Japanese Hot Bath in the Senior Population. Journal of the Japanese Society of Balneology, Climatology and Physical Medicine, 72, 31-35.

[13] Shigeomi, S., Sato, W., Enzan, K. and Yoshioka, N. (2001) Sudden Death during Bathing in the Elderly. JJAAM, 12, 109-120. http://dx.doi.org/10.3893/jjaam.12.109

[14] Schuessler, R.B., Grayson, T.M., Bromberg, B.I., Cox, J.L. and Boineau, J.P. (1992) Cholinergically Medicated Tachyarrhythmias Induced by a Single Extra Stimulus in the Isolated Canine Right Atrium. Circulation Research, 71, 1254-1267. http://dx.doi.org/10.1161/01.RES.71.5.1254

[15] Wijffels, M.C., Kirchhof, C.J., Dorland, R. and Allessie, M.A. (1995) Atrial Fibrillation Begets Atrial Fibrillation. A Study in Awake Chronically Instrumented Goats. Circulation, 92, 1954-1968. http://dx.doi.org/10.1161/01.CIR.92.7.1954

[16] Nattel, S. and Li, D. (2000) Ionic Remodeling in the Heart: Pathophysiological Significance and New Therapeutic Opportunities for Atrial Fibrillation. Circulation Research, 87, 440-447. http://dx.doi.org/10.1161/01.RES.87.6.440

[17] Yue, L., Feng, J., Gaspo, R., Li, G.R., Wang, Z. and Nattel, S. (1997) Ionic Remodeling Underlying Action Potential Changes in a Canine Model of Atrial Fibrillation. Circulation Research, 81, 512-525. http://dx.doi.org/10.1161/01.RES.81.4.512

[18] Wu, T.J., Yashima, M., Xie, F., Athill, C.A., Kim, Y.H., Fishbein, M.C., Qu, Z., Garfinkel, A., Weiss, J.N., Karagueuzian, H.S. and Chen, P.S. (1998) Role of Pectinate Muscle Bundles in the Generation and Maintenance of Intra-Atrial Reentry: Potential Implications for the Mechanism of Conversion between Atrial Fibrillation and Atrial Flutter. Circulation Research, 83, 448-462. http://dx.doi.org/10.1161/01.RES.83.4.448 